CERN-TH/97-23

\title{
A THEOREM ON THE REAL PART OF THE HIGH-ENERGY SCATTERING AMPLITUDE NEAR THE FORWARD DIRECTION
}

\author{
André MARTIN*) \\ Theoretical Physics Division, CERN \\ CH - 1211 Geneva 23 \\ and \\ LAPP*), B.P. 110 \\ F - 74941 Annecy Le Vieux \\ E-mail: martina@mail.cern.ch
}

\begin{abstract}
We show that if for fixed negative (physical) square of the momentum transfer $t$, the differential cross-section $\frac{d \sigma}{d t}$ tends to zero and if the total cross-section tends to infinity, when the energy goes to infinity, the real part of the even signature amplitude cannot have a constant sign near $t=0$.
\end{abstract}

*) URA 14-36 du CNRS, associée à L'Ecole Normale Supérieure de Lyon et à l'Université de Savoie.

CERN-TH/97-23

January 1997 
The measurement of the real part of the scattering amplitude in the forward direction is a crucial test of dispersion relations. It allows to get information on the total cross-section at higher energies [1], [2] and also it may be a crucial test of locality. A violation of dispersion relations might be the sign of new physics like for example the existence of extra compact dimensions [3].

To measure this real part, one has to use Coulomb interference, which is large at a very small but not zero momentum transfer. Most of the time people assume that in the region where the interference takes place, the ratio of the real to imaginary part, called $\rho$, is constant. For exceptions, see the papers of Kundrat and collaborators [4]. The present paper contains a theorem which constitutes a kind of warning against the dangers of this procedure, because we show that under assumptions which are satisfied by most existing models and seem to be compatible with experimental data on proton (anti)proton scattering, namely that $\frac{d \sigma}{d t}$ tends to zero for fixed negative $t$ and that $\sigma_{\text {total }}$ tends to infinity, the real part of the even signature amplitude cannot have a constant sign in a strip $-T<t \leq 0, s>s_{M}$, where $T$ is arbitrarily small and $s_{M}$ is arbitrarily large. Hence, $\rho$ cannot be constant because it is extremely difficult to make the imaginary part of the amplitude vanish exactly at the same place as the real part because of positivity constraints, and also because, from a practical point of view, the differential cross-section is not seen to vanish anywhere. The question is therefore if it is still admissible to neglect the variation of $\rho$ in a measurement of the real part by interference. In a simple case we shall show that the zero of the real part is more than 100 times further away than the place where the interference is maximum, but we have no certainty about this in general. Of course, in a specific model, like for instance the one of $\mathrm{Wu}$ and collaborators [5], there is no problem because one can test directly if $\rho(s, t)$ given by the model reproduces the interference curve, but it is unfortunate that one cannot get $\rho(s, t=0)$ without any theoretical prejudice.

We now come to Theorem I. Consider an amplitude $F(s, t, u)$ describing $A+B \rightarrow A+B$ and $A+\bar{B} \rightarrow A+\bar{B}$ reactions with

$$
s+t+u=2 M_{A}^{2}+2 M_{B}^{2},
$$

$s$, square of centre-of-mass energy in the $A+B \rightarrow A+B$ channel and $u$ in the $A+\bar{B} \rightarrow A+\bar{B}$ channel and $t=-2 k^{2}(1-\cos \theta), k$ centre-of-mass momentum and $\cos \theta$ scattering angle in the $\mathrm{AB}$ channel. If the amplitude is not symmetric in $s-u$ exchange, we symmetrize it to get the "even-signature" amplitude. Then we state:

\section{Theorem I}

If $F$ is $s-u$ crossing symmetric and if $\operatorname{Re} F$ has a constant sign (including zero) in the strip $s_{M}<s<\infty,-t<T \leq 0$ where $s_{M}$ is arbitrarily large and $T>0$ arbitrarily small, and if $\frac{F(s, t)}{s} \rightarrow 0$ for $-T<t<0,|F(s, t=0) / s|$ does not tend to $\infty$ for $s \rightarrow \infty$ and therefore by the optical theorem the total cross-section does not tend to infinity.

Theorem II

If $\frac{d \sigma}{d t}(A+B \rightarrow A+B)$ and $\frac{d \sigma}{d t}(A+\bar{B} \rightarrow A+\bar{B})$ tend to zero for $s \rightarrow \infty-T<t<0$ for some $T$ and if $\sigma_{\text {total }}(A B)$ and/or $\sigma_{\text {total }}(A \bar{B})$ tend to infinity for infinite energy, the real part of the 
even signature amplitude cannot have a constant sign in a strip $s_{M}<s<\infty,-T<t \leq 0$, for any $s_{M}$ and $T>0$.

Theorem II is an obvious consequence of Theorem I.

Proof of Theorem I

We work with a crossing symmetric amplitude in the exchange $s \leftrightarrow u$. For $-T<t \leq 0$ this amplitude satisfies twice subtracted dispersion relations (in fact this holds also for $-T<t<+R$, $R>0$ as a consequence of the axioms of local field theory and positivity [6]). It means that the amplitude is analytic in a twice cut plane. If we use the variable

$$
\begin{gathered}
z(s, t)=(s-u)^{2}=\left(2 s-2\left(M_{A}^{2}+M_{B}^{2}\right)+t\right)^{2}, \\
F(s, t, u)=G(z, t)
\end{gathered}
$$

$G$ is analytic in a once cut plane for $-T<t$ real $\leq 0$, with, in the simplest case of a "normal threshold" a cut beginning at

$$
z_{0}(t)=\left(4 M_{A} M_{B}+t\right)^{2}
$$

We can write an "inverse" dispersion relation for $G$. Define:

$$
H=\frac{G(z, t)}{\sqrt{z_{0}(t)-z}} .
$$

If $F(s, t) / s$ tends to zero for $t<0, s \rightarrow \infty, H$ satisfies an unsubtracted dispersion relation

$$
H(z, t)=-\frac{1}{\pi} \int_{z_{0}(t)}^{\infty} \frac{\operatorname{Re} G\left(z^{\prime}, t\right)}{\sqrt{z^{\prime}-z_{0}}\left(z^{\prime}-z\right)} d z .
$$

Suppose now that $\operatorname{Re} F(s, t)$ has a constant sign, say negative, for $s>s_{0},-T<t \leq 0$. $\operatorname{Re} G(z, t)$ has then also a constant negative sign for $-T<t \leq 0$ and $z>z_{M}(t)=\left[2\left(s_{M}-M_{A}^{2}-m_{B}^{2}\right)+t\right]^{2}$

We write $H$ as

$$
H=\hat{H}+\Delta
$$

where

$$
\hat{H}_{(z, t)}=\frac{1}{\pi} \int_{z_{M}(t)}^{\infty} \frac{-\operatorname{Re} G\left(z^{\prime}, t\right) d z^{\prime}}{\sqrt{z^{\prime}-z_{0}(t)}\left(z^{\prime}-z\right)}
$$

and

$$
\Delta(z, t)=\frac{1}{\pi} \int_{z_{0}(t)}^{z_{M}(t)} \frac{-\operatorname{Re} G\left(z^{\prime}, t\right) d z^{\prime}}{\sqrt{z^{\prime}-z_{0}(t)}\left(z^{\prime}-z\right)} .
$$

Notice that for $|z| \rightarrow \infty,|\Delta|$ is bounded by const $/|z|$.

$\hat{H}$ having a positive discontinuity is a "Herglotz" function, having no zeros in the complex plane. For $z<0$, it is positive and increasing. Hence

$$
0<\hat{H}(-x, t)<\hat{H}(0, t) \text { for }-T<t<0, \quad x>0
$$


so that $\hat{H}$ is uniformly bounded on the negative real axis.

Now we remark that $F(s, t)$ is analytic in $t$, for fixed $s$ in a circle $|t| \leq R$ [6], and hence, $G(z, t)$ and $H(z, t)$ are both analytic in a neighbourhood of $-R<t<+R$ for any complex $z$ and also for $z \leq 0$ (one must pay attention to the fact that the cuts move with $t$ ).

Concerning $\Delta(z, t)$, we can reexpress it as an integral over the original variables $s^{\prime}$ and $t$ over the range $s_{0}<s^{\prime}<s_{M}$, and reach again the conclusion that when $z$ is strictly outside the cut

$$
\left(2\left(s_{0}-M_{A}^{2}-M_{B}^{2}\right)-T\right)^{2}<z<\left(2\left(s_{M}-M_{A}^{2}-M_{B}^{2}\right)\right)^{2},
$$

$\Delta$ is analytic in $t$ in a neighbourhood of $-R<t<+R$.

Therefore, $\hat{H}$ is also analytic in $t$, for fixed $z,-\infty<z<0$, in a neighbourhood of $-R<t<+R$. Hence inequality (9) which was established for $t$ strictly negative also holds by continuity for $t=0$ :

$$
0 \leq \hat{H}(-x, 0) \leq \hat{H}(0,0)
$$

Now suppose that $\sigma_{\text {total }} \rightarrow \infty$ for $s \rightarrow \infty$. This means that $|F(s, t=0) / s| \rightarrow \infty$, and hence $|H(z, t=0)| \rightarrow \infty$ for $z \rightarrow+\infty$ on both sides of the cut.

Since $|\Delta(z, t=0)|$ can be bounded by const $/|z|$, this also means that

$$
|\hat{H}(z, t=0)| \rightarrow \infty
$$

But $-1 / \hat{H}(z, t=0)$ is also a Herglotz function and, from (11)

$$
\left|\frac{1}{\hat{H}(z, t=0)}\right| \rightarrow 0,
$$

for $z \rightarrow+\infty$, on both sides of the cut. By the Phragmén-Lindelöf theorem $1 / \hat{H}(z, t=0)$ also tends to zero for $|z| \rightarrow \infty$ in any complex direction. This contradicts the fact that $\hat{H}(-x, t=0)$ is uniformly bounded for $x>0$, and therefore $\sigma_{t}(s)$ cannot tend to infinity.

Strictly speaking this implies that $\sigma_{t}(s)$ has a finite least lower limit for $s \rightarrow \infty$. One can probably make a more refined statement on a smoothed $\sigma_{t}$. Of course, if we believe that $\sigma_{t}$ is monotonous beyond a certain energy, it means that $\sigma_{t}$ is bounded.

As we said, Theorem II is a direct consequence of Theorem I. In practice, Theorem II is the most relevant, because there are clear indications, in the case of proton-antiproton scattering that $\sigma_{t}$ is increasing [7] and, comparing for instance the ISR and sp $\bar{p}$ s data [7], that $\frac{d \sigma}{d t}$ decreases at least for $t<-0.4 \mathrm{GeV}^{2}$, and it seems that the decrease occurs for smaller and smaller $|t|$ when the energy increases.

The simplest way to satisfy theorem II is to have a curve in the $s-t$ plane where the real part changes sign:

$$
t=-f(s), \quad f(s)>0
$$


such that $f(s) \rightarrow 0$ for $s \rightarrow \infty$, but this is not the only possibility. We could also have an infinite sequence of bubbles in the $s-t$ plane approaching $t=0$ for $s \rightarrow \infty$ in which $\operatorname{Re} F$ would have an opposite sign. The implication of the theorem for the individual $A B \rightarrow A B$ and $A \bar{B} \rightarrow A \bar{B}$ amplitudes is that their real parts cannot have both the same constant sign.

Now, is the theorem a surprise? Not really if we look at simple-minded examples. What is a surprise is its generality.

Examples can be built by taking

$$
|F(s, t)| \sim s^{1+\lambda t}(\log s)^{\gamma}
$$

with $0<\gamma \leq 1, \lambda>0$. F/s goes to zero for $t<0 \quad s \rightarrow \infty$, and $\sigma_{t} \sim(\log s)^{\gamma}$.

Such examples do not manifestly violate $s$ channel unitarity nor polynomial boundedness for fixed complex $t$ (for $\sigma_{t} \sim(\log s)^{\gamma}, 1<\gamma<2$ model building is more tricky while the extreme case $\gamma=2$ is easy to handle [8]). By standard techniques we can make this amplitude even under crossing by replacing it by

$$
F(s, t)=i C e^{\frac{-i \lambda t \pi}{2}} s^{1+\lambda t}\left(\log s-\frac{i \pi}{2}\right)^{\gamma},
$$

with $C$ real $>0$.

For small $t$, we see that the real part changes sign for

$$
t=-\frac{\gamma}{\lambda \log s}
$$

The special case $\gamma=1$ corresponds to what is called "geometrical scaling". Indeed, $\phi=F(s, t) / F(s, 0)$ is just a function of $t \log s$, i.e., of $\tau=t \sigma_{t}$, since $\sigma_{t} \sim \log s$. In that special case the real part, according to an old theorem of the author [9], is found proportional to $\frac{d}{d \tau}(\tau \phi(\tau))$ and we check that this gives us precisely a zero at

$$
t=-\frac{1}{\lambda \log s}
$$

However, for $\gamma<1$ this is no longer true and this shows how dangerous is the abusive use of this "magic" formula.

We would like now to return to the question of how our result affects the measurement of the real part. In general, it is difficult to say, but, in the special case of geometrical scaling the same function determines the point of maximal interference and the location of the zero of the real part. Indeed the complete amplitude, including the Coulomb term is given (with some oversimplifications !) by [8]

$$
F=\frac{\alpha s}{2 t}+F_{H}(s, t),
$$

where $F_{H}$ reduces to $s \sigma_{\text {total }}(\rho+i) / 16 \pi$ at $t=0$. We can rewrite $F$ as

$$
\frac{\alpha s}{2 t}+\frac{s \sigma_{t}(\rho(0)+i)}{16 \pi} \phi\left(t \sigma_{t}\right)
$$


and the relative maximum interference occurs near

$$
\psi\left(t \sigma_{t}\right)=8 \pi \alpha
$$

where

$$
\psi(\tau)=\tau \phi(\tau)
$$

while the zero of the real part is given by

$$
\psi^{\prime}=0
$$

It happens that in practice the point of maximum relative interference for proton-(anti)proton scattering corresponds to $t \simeq 10^{-3} \mathrm{GeV}^{2}$ while, if we approximate the diffraction peak by $\exp B t$, the zero of $\psi^{\prime}$ is at $t=(B / 2)^{-1} \cong 0.13(\mathrm{GeV})^{2}$ at $\sqrt{s}=1 \mathrm{TeV}$ [7], [4], so that the situation is not too bad. However, outside the geometrical scaling regime (which, incidentally does not seem to apply to proton-antiproton scattering at energies above the ISR range, since the ratio of the elastic to total cross-sections increases instead of being constant [7]), Eq. (16) shows that the zero can be at a different place. In the model of $\mathrm{Wu}$ et al. [5] the first zero of Re $F$ is at $t=-0.32 \mathrm{GeV}^{2}$ for $\sqrt{s}=1 \mathrm{TeV}$, according to C. Bourrely.

Finally, for completeness we would like to present the counterpart of Theorem I for the odd signature amplitude:

\section{Theorem III}

If, in the strip, $-T<t<0, s>s_{M}$, the difference of the imaginary parts of the amplitudes $A B \rightarrow A B$ and $A \bar{B} \rightarrow A \bar{B}$ has a constant sign, and if $\frac{d \sigma}{d t} \rightarrow 0$ for both reactions, the difference of the total cross-sections $\sigma_{A B}-\sigma_{A \bar{B}}$ does not tend to infinity.

This means that the "maximal odderon" [10] is excluded under the above conditions. We leave the proof as an exercise to the reader.

\section{Acknowledgements}

I would like to thank Jan Fischer, Maurice Haguenauer and Claude Bourrely for very stimulating discussions. 


\section{References}

[1] U. Amaldi et al., Phys.Lett. 66B (1977) 390.

[2] C. Augier et al. (UA4 Collaboration), Phys.Lett. B316 (1993) 448.

[3] N.N. Khuri, in Seventh "Rencontres de Blois" on Elastic and Diffractive Scattering, Blois 1995, Editions Frontiéres, BP. 33, Gif sur Yvette (1996), p. 471.

[4] V. Kundrat and M. Lokajiček, Phys.Rev. D31 (1985) 1045, Phys.Lett. B232 (1989) 263, and 1996 Preprint, Institute of Physics ASCR, to appear in Phys.Rev. D.

[5] H. Cheng and T.T. Wu,Phys.Rev.Lett. 24 (1970) 1456, and

C. Bourrely, J. Soffer and T.T. Wu, Ref. [3], p. 15.

[6] A. Martin, Nuovo Cimento 42 (1966) 930.

[7] A. Martin and G. Matthiae, in Proton-Antiproton Physics, G. Altarelli and L. Di Lella Eds., World Scientific, Singapore (1989), pp. 45-84, and

M. Haguenauer, Private communication;

G. Matthiae, Rep.Progr.Phys. 57 (1994) 743.

[8] G. Auberson, A. Martin and T. Kinoshita, Phys.Rev. D3 (1971) 3185.

[9] A. Martin, Lett. Nuovo Cimento 7 (1973) 811.

[10] L. Łukaszuk and B. Nicolescu, Lett. Nuovo Cimento 8 (1973) 405. 